\title{
Relations for product moments and covariances of $k$ th records from discrete distributions
}

\author{
Krzysztof Jasiński ${ }^{1}$
}

Received: 1 April 2017 / Published online: 30 November 2017

(C) The Author(s) 2017. This article is an open access publication

\begin{abstract}
The aim of this paper is to establish recurrence relations satisfied by product moments and covariances of $k$ th records arising from discrete distributions. They will be evaluated for geometric underlying distribution. Then we use these results to obtain formulas for correlation coefficients of geometric $k$ th records. We consider all three known types of $k$ th records: strong, ordinary, and weak.
\end{abstract}

Keywords Product moments · Covariances · Correlation coefficients · Strong, ordinary and weak $k$ th records $\cdot$ Geometric distribution

\section{Introduction}

Let $X_{1}, X_{2}, \ldots$ be a sequence of independent and identically distributed (iid) random variables (rv's) with cumulative distribution function (cdf) $F$. Let $X_{1: n} \leq \cdots \leq X_{n: n}$ stand for the respective order statistics of a random sample $\left(X_{1}, X_{2}, \ldots, X_{n}\right)$. For positive integer $k$, we define occurrence times $\left(T_{n}^{(k)}, n \geq 0\right)$ and values of $k$ th records $\left(R_{n}^{(k)}, n \geq 0\right)$ as follows:

$$
\begin{aligned}
& T_{0}^{(k)}=k, \quad T_{n+1}^{(k)}=\min \left\{j>T_{n}^{(k)}: X_{j}>X_{T_{n}^{(k)}-k+1: T_{n}^{(k)}}\right\}, \quad n \geq 0, \\
& R_{n}^{(k)}=X_{T_{n}^{(k)}-k+1: T_{n}^{(k)}}, \quad n \geq 0 .
\end{aligned}
$$

Krzysztof Jasiński krzys@mat.umk.pl

1 Faculty of Mathematics and Computer Science, Nicolaus Copernicus University, ul. Chopina 12/18, 87-100 Toruń, Poland 
Note that for $k=1$ the sequences of 1 st record times $\left(T_{n}^{(1)}, n \geq 0\right)$ and 1 st records $\left(R_{n}^{(1)}, n \geq 0\right)$ reduce to the sequences of record times $\left(T_{n}, n \geq 0\right)$ and record values $\left(R_{n}, n \geq 0\right)$, respectively. For a deeper discussion of records and $k$ th records we refer the reader to the monographs of Arnold et al. (1998) and Nevzorov (2001).

If the underlying distribution is continuous, then the sequence of $k$ th records from a cdf $F$ is distributed as the sequence of records from a cdf $F_{1: k}=1-(1-F)^{k}$ :

$$
\left\{R_{n}^{(k)}(F), n \geq 0\right\} \stackrel{\mathrm{d}}{=}\left\{R_{n}\left(F_{1: k}\right), n \geq 0\right\}, \quad k=1,2, \ldots,
$$

where $\stackrel{d}{=}$ denotes equality in distribution; see Nevzorov (2001, Theorem 22.6). This fact implies that in the continuous case distributional properties of $k$ th records can be derived in a direct manner from those of records. However, there are some results concerning $k$ th records which do not follow from the corresponding ones for records. An example of such non-trivial results are various bounds on moments of $k$ th records, see for example Raqab and Rychlik (2002, 2004, 2011), Klimczak and Rychlik (2004, 2005), Charalambides and Rychlik (2008), Goroncy and Rychlik (2011), Jasiński (2016), Kozyra and Rychlik (2017).

The situation becomes more complicated for discrete distributions. López-Blázquez et al. (2005) and Dembińska and López-Blázquez (2005b) showed that then (1.1) is not valid. Moreover, in the last-mentioned paper definitions of strong and weak $k$ th records were introduced.

Definition 1 The sequences of strong $k$ th record times $\left(L_{n}^{(k)}, n \geq 0\right)$ and strong $k$ th records $\left(S_{n}^{(k)}, n \geq 0\right)$ are given by:

$$
\begin{aligned}
& L_{0}^{(k)}=1, \quad L_{n+1}^{(k)}=\min \left\{j>L_{n}^{(k)}: X_{j: j+k-1}>X_{L_{n}^{(k)}: L_{n}^{(k)}+k-1}\right\}, \quad n \geq 0, \\
& S_{n}^{(k)}=X_{L_{n}^{(k)}: L_{n}^{(k)}+k-1}, \quad n \geq 0 .
\end{aligned}
$$

Definition 2 The sequences of weak $k$ th record times $\left(U_{n}^{(k)}, n \geq 0\right)$ and weak $k$ th records $\left(W_{n}^{(k)}, n \geq 0\right)$ are given by:

$$
\begin{aligned}
& U_{0}^{(k)}=k, \quad U_{n+1}^{(k)}=\min \left\{j>U_{n}^{(k)}: X_{j} \geq X_{U_{n}^{(k)}-k+1: U_{n}^{(k)}}\right\}, \quad n \geq 0, \\
& W_{n}^{(k)}=X_{U_{n}^{(k)}-k+1: U_{n}^{(k)}}, \quad n \geq 0 .
\end{aligned}
$$

When $k=1$, the definition of 1 st weak records amounts to the definition of weak records $\left(W_{n}, n \geq 0\right)$, which were introduced by Vervaat (1973) and investigated among others by Stepanov (1992, 1993), Wesołowski and Ahsanullah (2001), Stepanov et al. (2003), Dembińska and Stepanov (2006), Danielak and Dembińska (2007a,b), Ahsanullah and Aliev (2011), Karczewski and Wesołowski (2017).

Properties of weak $k$ th records, (ordinary) $k$ th records and strong $k$ th records are extensively studied in the literature. We refer to Dembińska and López-Blázquez (2005a), Dembińska (2007a,b, 2008), Dembińska and Danielak (2008) and Oncel and Aliev (2016). However, many problems concerning $k$ th records from discrete 
distributions are still open. The aim of this paper is to fill this gap in the literature. Precisely, we derive recurrence relations satisfied by product moments of $k$ th records arising from discrete distributions. These results together with the corresponding ones for single moments obtained by Dembińska and Danielak (2008) allow us to establish direct formulas for covariances and correlation coefficients between $R_{m}^{(k)}$ and $R_{n}^{(k)}$, $W_{m}^{(k)}$ and $W_{n}^{(k)}$ or $S_{m}^{(k)}$ and $S_{n}^{(k)}, 0 \leq m<n$ in some specific cases, when the underlying distribution is geometric.

\section{Basic properties of discrete $k$ th records}

In this section, we recall some results concerning the distribution of discrete $k$ th records, which can be found in Dembińska and López-Blázquez (2005b) and Dembińska and Danielak (2008). They will be useful in Sect. 3.

From now on we assume that $X_{1}, X_{2}, \ldots$ are iid rv's with support on non-negative integers, probability mass function $p_{j}=\mathrm{P}\left(X_{1}=j\right)$ and $q_{j}=\mathrm{P}\left(X_{1} \geq j\right), j \geq 0$.

The following lemma describes how the distributional properties of discrete strong $k$ th records can be derived from properties of records $\left(R_{n}, n \geq 0\right)$.

Lemma 1 For any $k=1,2, \ldots$ the sequence of strong $k$ th records $\left(S_{n}^{(k)}, n \geq 0\right)$ from a cdf $F$ is identical in distribution to a sequence of records $\left(R_{n}, n \geq 0\right)$ from a $c d f F_{1: k}=1-(1-F)^{k}$ :

$$
\left\{S_{n}^{(k)}(F), n \geq 0\right\} \stackrel{\mathrm{d}}{=}\left\{R_{n}\left(F_{1: k}\right), n \geq 0\right\} .
$$

For ordinary and weak $k$ th records the analogs of (2.1) are not valid. To analyze their properties, it is convenient to define new rv's $\eta_{m}^{(k)}, \xi_{m}^{(k)}, m=0,1, \ldots, k=1,2, \ldots$ as follows:

$$
\begin{aligned}
& \eta_{m}^{(k)}=s \text { if there are exactly } s k \text { th records that are equal to } m, \\
& \xi_{m}^{(k)}=s \text { if there are exactly } s \text { weak } k \text { th records that are equal to } m .
\end{aligned}
$$

To pass from dependent ordinary $k$ th records and weak $k$ th records to independent rv's we use the lemma and representation given below.

Lemma 2 For any $k=1,2, \ldots$ and $m=0,1, \ldots$ :

(a) the $r v$ 's $\eta_{0}^{(k)}, \eta_{1}^{(k)}, \ldots$ are independent and $\eta_{m}^{(k)}$ has a binomial distribution $b\left(k, \frac{p_{m}}{q_{m}}\right)$ with

$$
P\left(\eta_{m}^{(k)}=s\right)=\left(\begin{array}{l}
k \\
s
\end{array}\right)\left(\frac{p_{m}}{q_{m}}\right)^{s}\left(1-\frac{p_{m}}{q_{m}}\right)^{k-s}, \quad s=0,1, \ldots, k
$$


(b) the rv's $\xi_{0}^{(k)}, \xi_{1}^{(k)}, \ldots$ are independent and $\xi_{m}^{(k)}$ has a negative binomial distribution $n b\left(k, 1-\frac{p_{m}}{q_{m}}\right)$ with

$$
P\left(\xi_{m}^{(k)}=s\right)=\left(\begin{array}{c}
s+k-1 \\
s
\end{array}\right)\left(\frac{p_{m}}{q_{m}}\right)^{s}\left(1-\frac{p_{m}}{q_{m}}\right)^{k}, \quad s=0,1, \ldots
$$

Representation 1 For $m=1,2, \ldots, n=0,1, \ldots$, and $k=1,2, \ldots$ :

$$
\begin{aligned}
& \left\{R_{n}^{(k)}>m\right\}=\left\{\eta_{0}^{(k)}+\eta_{1}^{(k)}+\cdots+\eta_{m}^{(k)} \leq n\right\}, \\
& \left\{W_{n}^{(k)}>m\right\}=\left\{\xi_{0}^{(k)}+\xi_{1}^{(k)}+\cdots+\xi_{m}^{(k)} \leq n\right\} .
\end{aligned}
$$

Now, we are in a position to derive formulas for product moments and covariances of $k$ th records, weak $k$ th records and strong $k$ th records. All these results will be evaluated for the geometric underlying distribution $g e(p)$ with $p_{j}=p q^{j}, j=0,1, \ldots$, where $q=1-p \in(0,1)$. We will use the recurrence relations for the first moment of any discrete ordinary and weak $k$ th records established by Dembińska and Danielak (2008). They showed that for $k=1,2, \ldots$

$$
\begin{gathered}
\mathrm{E}\left(R_{n}^{(k)}\right)= \begin{cases}\sum_{m_{1}=0}^{\infty} q_{m_{1}+1}^{k}, & n=0, \\
\mathrm{E}\left(R_{n-1}^{(k)}\right)+\sum_{m_{1}=0}^{\infty} \mathrm{P}\left(N_{m_{1}}^{(k)}=n\right), & n \geq 1,\end{cases} \\
\mathrm{E}\left(W_{n}^{(k)}\right)= \begin{cases}\sum_{m_{1}=0}^{\infty} q_{m_{1}+1}^{k}, & n=0, \\
\mathrm{E}\left(W_{n-1}^{(k)}\right)+\sum_{m_{1}=0}^{\infty} \mathrm{P}\left(M_{m_{1}}^{(k)}=n\right), & n \geq 1,\end{cases}
\end{gathered}
$$

with $N_{m}^{(k)}=\eta_{0}^{(k)}+\eta_{1}^{(k)} \cdots+\eta_{m}^{(k)}$ and $M_{m}^{(k)}=\xi_{0}^{(k)}+\xi_{1}^{(k)} \cdots+\xi_{m}^{(k)}$ respectively, where the rv's $\xi_{i}^{(k)}$ and $\eta_{i}^{(k)}$ are described in Lemma 2. Moreover, in the geometric case, they obtained simple expressions for means and variances as follows

$$
\mathrm{E}\left(R_{n}^{(k)}\right)=\sum_{j=0}^{n} \frac{p^{j}}{j !}\left(\frac{1}{1-q^{k}}\right)^{(j)}-1, \quad \mathrm{E}\left(W_{n}^{(k)}\right)=q \sum_{j=0}^{n} \frac{p^{j}}{j !}\left(\frac{q^{k+j-1}}{1-q^{k}}\right)^{(j)},
$$

$\operatorname{Var}\left(R_{0}^{(k)}\right)=\operatorname{Var}\left(W_{0}^{(k)}\right)=\frac{q^{k}}{\left(1-q^{k}\right)^{2}}$,

where $(f(q))^{(j)}$ stands for the $j$ th derivative of the function $f(q)$. In the next section, (2.4) and (2.5) will be used to get covariances and correlation coefficients for geometric $k$ th records. 


\section{Product moments and covariances}

To derive formulas expressing product moments of $k$ th records arising from discrete distributions we will use a multivariate extension of Hoeffding's (1940) lemma. Block and Fang (1988) showed that if $Z_{1}, \ldots, Z_{d}$ are non negative rv's then

$$
\mathrm{E}\left(Z_{1} \ldots Z_{d}\right)=\int_{0}^{\infty} \cdots \int_{0}^{\infty} P\left(Z_{1}>x_{1}, \ldots, Z_{d}>x_{d}\right) d x_{1} \ldots d x_{d} \text {. }
$$

If $Z_{1}, \ldots, Z_{d}$ take values in the set of non-negative integers, the above expression can be written as

$$
\mathrm{E}\left(Z_{1} \ldots Z_{d}\right)=\sum_{m_{1}=0}^{\infty} \cdots \sum_{m_{d}=0}^{\infty} P\left(Z_{1}>m_{1}, \ldots, Z_{d}>m_{d}\right) .
$$

In particular, we obtain well-known formulas for the first two moments of a single rv $Z_{1}$ :

$$
\mathrm{E} Z_{1}=\sum_{m=0}^{\infty} P\left(Z_{1}>m\right) \quad \text { and } \quad \mathrm{E} Z_{1}^{2}=\sum_{m=0}^{\infty}(2 m+1) P\left(Z_{1}>m\right)
$$

\section{$3.1 k$ th records}

We begin with a general result on ordinary $k$ th records. We use the convention that $R_{-1}^{(k)}=0$ for $k \geq 1$.

Theorem 1 For any $k=1,2, \ldots$, and $0 \leq m<n$ :

$$
\begin{aligned}
\mathrm{E}\left(R_{m}^{(k)} R_{n}^{(k)}\right)= & \mathrm{E}\left(R_{m-1}^{(k)} R_{n}^{(k)}\right)+\sum_{m_{1}=0}^{\infty} \mathrm{P}\left(N_{m_{1}}^{(k)}=m\right) \\
& \times \sum_{m_{2}=m_{1}}^{\infty} \sum_{j=0}^{n-m} \mathrm{P}\left(N_{m_{2}}^{(k)}-N_{m_{1}}^{(k)}=j\right)+\sum_{m_{1}=0}^{\infty} m_{1} \mathrm{P}\left(N_{m_{1}}^{(k)}=m\right) .
\end{aligned}
$$

Proof Using (3.1) for $d=2$ and Representation 1 we get

$$
\begin{aligned}
\mathrm{E}\left(R_{m}^{(k)} R_{n}^{(k)}\right)= & \sum_{m_{1}=0}^{\infty} \sum_{m_{2}=0}^{\infty} \mathrm{P}\left(R_{m}^{(k)}>m_{1}, R_{n}^{(k)}>m_{2}\right) \\
= & \sum_{m_{1}=0}^{\infty} \sum_{m_{2}=0}^{\infty} \mathrm{P}\left(N_{m_{1}}^{(k)} \leq m, N_{m_{2}}^{(k)} \leq n\right) \\
= & \sum_{m_{1}=0}^{\infty} \sum_{m_{2}=0}^{\infty} \mathrm{P}\left(N_{m_{1}}^{(k)} \leq m-1, N_{m_{2}}^{(k)} \leq n\right) \\
& +\sum_{m_{1}=0}^{\infty} \sum_{m_{2}=0}^{\infty} \mathrm{P}\left(N_{m_{1}}^{(k)}=m, N_{m_{2}}^{(k)} \leq n\right)
\end{aligned}
$$




$$
\begin{aligned}
= & \mathrm{E}\left(R_{m-1}^{(k)} R_{n}^{(k)}\right)+\sum_{m_{1}=0}^{\infty} \sum_{m_{2}=m_{1}}^{\infty} \mathrm{P}\left(N_{m_{1}}^{(k)}=m, N_{m_{2}}^{(k)} \leq n\right) \\
& +\sum_{m_{1}=1}^{\infty} \sum_{m_{2}=0}^{m_{1}-1} \mathrm{P}\left(N_{m_{1}}^{(k)}=m, N_{m_{2}}^{(k)} \leq n\right) .
\end{aligned}
$$

When $m_{2}<m_{1}$, the inequality $N_{m_{2}}^{(k)}=\eta_{0}^{(k)}+\ldots+\eta_{m_{2}}^{(k)} \leq m<n$ implies $\mathrm{P}\left(N_{m_{1}}^{(k)}=\right.$ $\left.m, N_{m_{2}}^{(k)} \leq n\right)=\mathrm{P}\left(N_{m_{1}}^{(k)}=m\right)$. Thus

$$
\sum_{m_{1}=1}^{\infty} \sum_{m_{2}=0}^{m_{1}-1} \mathrm{P}\left(N_{m_{1}}^{(k)}=m, N_{m_{2}}^{(k)} \leq n\right)=\sum_{m_{1}=0}^{\infty} m_{1} \mathrm{P}\left(N_{m_{1}}^{(k)}=m\right)
$$

By similar arguments, for $m_{2} \geq m_{1}$, we get

$$
\mathrm{P}\left(N_{m_{1}}^{(k)}=m, N_{m_{2}}^{(k)} \leq n\right)=\sum_{j=0}^{n-m} \mathrm{P}\left(N_{m_{1}}^{(k)}=m, N_{m_{2}}^{(k)}-N_{m_{1}}^{(k)}=j\right) .
$$

Hence, the independence of rv's $N_{m_{1}}^{(k)}$ and $N_{m_{2}}^{(k)}-N_{m_{1}}^{(k)}$ implies

$$
\mathrm{P}\left(N_{m_{1}}^{(k)}=m, N_{m_{2}}^{(k)} \leq n\right)=\mathrm{P}\left(N_{m_{1}}^{(k)}=m\right) \sum_{j=0}^{n-m} \mathrm{P}\left(N_{m_{2}}^{(k)}-N_{m_{1}}^{(k)}=j\right)
$$

Now, applying (3.3) and (3.4) to (3.2), we complete the proof.

Theorem 1 and formula (2.2) give the following recurrence relations for covariances between $k$ th records.

Corollary 1 For any $k=1,2, \ldots$, and $0 \leq m<n$ :

$$
\begin{aligned}
\operatorname{Cov}\left(R_{m}^{(k)}, R_{n}^{(k)}\right)= & \operatorname{Cov}\left(R_{m-1}^{(k)}, R_{n}^{(k)}\right) \\
& +\sum_{m_{1}=0}^{\infty} \mathrm{P}\left(N_{m_{1}}^{(k)}=m\right) \sum_{m_{2}=m_{1}}^{\infty} \sum_{j=0}^{n-m} \mathrm{P}\left(N_{m_{2}}^{(k)}-N_{m_{1}}^{(k)}=j\right) \\
& +\sum_{m_{1}=0}^{\infty}\left(m_{1}-\mathrm{E}\left(R_{n}^{(k)}\right)\right) \mathrm{P}\left(N_{m_{1}}^{(k)}=m\right) .
\end{aligned}
$$

The geometric distribution seems to be the only one for which the recurrence relations given in Theorem 1 and Corollary 1 lead to simple expressions.

Theorem 2 Assume that the parent distribution is geometric ge $(p)$. Then for $k=$ $1,2, \ldots$ and $0 \leq m<n$ : 


$$
\mathrm{E}\left(R_{m}^{(k)} R_{n}^{(k)}\right)= \begin{cases}\frac{q^{k}}{1-q^{k}} \sum_{j=0}^{n} \frac{p^{j}}{j !}\left(\frac{1}{1-q^{k}}\right)^{(j)}+\frac{q^{2 k}}{\left(1-q^{k}\right)^{2}}, & m=0, \\ \mathrm{E}\left(R_{m-1}^{(k)} R_{n}^{(k)}\right)+\frac{p^{m}}{m !}\left[\left(\frac{1}{1-q^{k}}\right)^{(m)}\left(\frac{m}{k}-1+\sum_{j=0}^{n-m} \frac{p^{j}}{j !}\left(\frac{1}{1-q^{k}}\right)^{(j)}\right)+\frac{q}{k}\left(\frac{1}{1-q^{k}}\right)^{(m+1)}\right], & m \geq 1,\end{cases}
$$

and

$$
\operatorname{Cov}\left(R_{m}^{(k)}, R_{n}^{(k)}\right)=\left\{\begin{array}{lr}
\operatorname{Var}\left(R_{0}^{(k)}\right), & m=0, \\
\operatorname{Cov}\left(R_{m-1}^{(k)}, R_{n}^{(k)}\right)+\frac{p^{m}}{m !}\left[\left(\frac{1}{1-q^{k}}\right)^{(m)}\left(\frac{m}{k}-\sum_{j=n-m+1}^{n} \frac{p^{j}}{j !}\left(\frac{1}{1-q^{k}}\right)^{(j)}\right)\right. & \\
\left.+\frac{q}{k}\left(\frac{1}{1-q^{k}}\right)^{(m+1)}\right], & m \geq 1,
\end{array}\right.
$$

and $\operatorname{Var}\left(R_{0}^{(k)}\right)$ is given by (2.5).

Proof In the geometric case, the rv's $\eta_{i}^{(k)}, i=0,1, \ldots$ are not only independent, but also have the same binomial distribution $b(k, p)$. Hence, $N_{m_{1}}^{(k)}$ has a binomial distribution with parameters $\left(m_{1}+1\right) k$ and $p$. With the convention $\left(\begin{array}{l}a \\ b\end{array}\right)=0$ for $a<b$, it follows that

$$
\begin{aligned}
\sum_{m_{1}=0}^{\infty} m_{1} \mathrm{P}\left(N_{m_{1}}^{(k)}=m\right)= & \left(\frac{p}{q}\right)^{m} \sum_{m_{1}=0}^{\infty} m_{1}\left(\begin{array}{c}
\left(m_{1}+1\right) k \\
m
\end{array}\right) q^{\left(m_{1}+1\right) k} \\
= & \left(\frac{p}{q}\right)^{m}\left[\sum_{m_{1}=1}^{\infty} m_{1}\left(\begin{array}{c}
m_{1} k \\
m
\end{array}\right) q^{m_{1} k}-\sum_{m_{1}=1}^{\infty}\left(\begin{array}{c}
m_{1} k \\
m
\end{array}\right) q^{m_{1} k}\right] \\
= & \left(\frac{p}{q}\right)^{m}\left[\frac{q}{k} \sum_{m_{1}=1}^{\infty} m_{1} k\left(\begin{array}{c}
m_{1} k \\
m
\end{array}\right) q^{m_{1} k-1}\right. \\
& \left.-\sum_{m_{1}=0}^{\infty}\left(\begin{array}{c}
m_{1} k \\
m
\end{array}\right) q^{m_{1} k}+I_{\{m=0\}}\right]
\end{aligned}
$$

where $I_{\{.\}}$is the indicator function such as $I_{\{m=0\}}=1$ if $m=0$ and $I_{\{m=0\}}=0$ otherwise. Using the formulas

$$
\begin{aligned}
\sum_{m_{1}=0}^{\infty}\left(\begin{array}{c}
m_{1} k \\
m
\end{array}\right) q^{m_{1} k} & =\frac{q^{m}}{m !} \sum_{m_{1}=0}^{\infty}\left(m_{1} k\right)\left(m_{1} k-1\right) \ldots\left(m_{1} k-m+1\right) q^{m_{1} k-m} \\
& =\frac{q^{m}}{m !}\left(\sum_{m_{1}=0}^{\infty} q^{m_{1} k}\right)^{(m)} \\
& =\frac{q^{m}}{m !}\left(\frac{1}{1-q^{k}}\right)^{(m)}
\end{aligned}
$$


and

$$
\begin{aligned}
\sum_{m_{1}=1}^{\infty} m_{1} k\left(\begin{array}{c}
m_{1} k \\
m
\end{array}\right) q^{m_{1} k-1} & =\left(\frac{q^{m}}{m !}\left(\frac{1}{1-q^{k}}\right)^{(m)}\right)^{(1)} \\
& =\frac{q^{m-1}}{m !}\left[m\left(\frac{1}{1-q^{k}}\right)^{(m)}+q\left(\frac{1}{1-q^{k}}\right)^{(m+1)}\right],
\end{aligned}
$$

for $m \geq 0$, after some algebra, we obtain

$$
\sum_{m_{1}=1}^{\infty} m_{1} \mathrm{P}\left(N_{m_{1}}^{(k)}=m\right)=\frac{p^{m}}{k m !}\left[(m-k)\left(\frac{1}{1-q^{k}}\right)^{(m)}+q\left(\frac{1}{1-q^{k}}\right)^{(m+1)}+k I_{\{m=0\}}\right] \text {. }
$$

Further, notice that $N_{m_{2}}^{(k)}-N_{m_{1}}^{(k)}$ has a binomial distribution with parameters $\left(m_{2}-m_{1}\right) k$ and $p$. Consequently, by (3.7) we have

$$
\begin{aligned}
& \sum_{m_{2}=m_{1}}^{\infty} \sum_{j=0}^{n-m} \mathrm{P}\left(N_{m_{2}}^{(k)}-N_{m_{1}}^{(k)}=j\right) \\
& =\sum_{j=0}^{n-m}\left(\frac{p}{q}\right)^{j} \sum_{m_{2}=0}^{\infty}\left(\begin{array}{c}
m_{2} k \\
j
\end{array}\right) q^{m_{2} k}=\sum_{j=0}^{n-m} \frac{p^{j}}{j !}\left(\frac{1}{1-q^{k}}\right)^{(j)} .
\end{aligned}
$$

This implies

$$
\begin{gathered}
\sum_{m_{1}=0}^{\infty} \mathrm{P}\left(N_{m_{1}}^{(k)}=m\right) \sum_{m_{2}=m_{1}}^{\infty} \sum_{j=0}^{n-m} \mathrm{P}\left(N_{m_{2}}^{(k)}-N_{m_{1}}^{(k)}=j\right) \\
=\left(\frac{p}{q}\right)^{m} \sum_{j=0}^{m-m} \frac{p^{j}}{j !}\left(\frac{1}{1-q^{k}}\right)^{(j)} \sum_{m_{1}=1}^{\infty}\left(\begin{array}{c}
m_{1} k \\
m
\end{array}\right) q^{m_{1} k} .
\end{gathered}
$$

Using again (3.7), we can rewrite the last series as

$$
\sum_{m_{1}=1}^{\infty}\left(\begin{array}{c}
m_{1} k \\
m
\end{array}\right) q^{m_{1} k}=\left[\frac{q^{m}}{m !}\left(\frac{1}{1-q^{k}}\right)^{(m)}-I_{\{m=0\}}\right] .
$$

Hence

$$
\begin{aligned}
& \sum_{m_{1}=0}^{\infty} \mathrm{P}\left(N_{m_{1}}^{(k)}=m\right) \sum_{m_{2}=m_{1}}^{\infty} \sum_{j=0}^{n-m} \mathrm{P}\left(N_{m_{2}}^{(k)}-N_{m_{1}}^{(k)}=j\right) \\
& = \begin{cases}\frac{q^{k}}{1-q^{k}} \sum_{j=0}^{n} \frac{p^{j}}{j !}\left(\frac{1}{1-q^{k}}\right)^{(j)}, & m=0, \\
\frac{p^{m}}{m !}\left(\frac{1}{1-q^{k}}\right) \sum_{j=0}^{(m)} \frac{p^{j}}{j !}\left(\frac{1}{1-q^{k}}\right)^{(j)}, & m \geq 1 .\end{cases}
\end{aligned}
$$


Combining the above equality with (3.8) and Theorem 1, we get (3.5).

Now, (3.5) and (2.4) give immediately (3.6). Indeed, for $m=0$ we obtain

$$
\begin{aligned}
\operatorname{Cov}\left(R_{0}^{(k)}, R_{n}^{(k)}\right)= & \mathrm{E}\left(R_{0}^{(k)} R_{n}^{(k)}\right)-\mathrm{E}\left(R_{0}^{(k)}\right) \mathrm{E}\left(R_{n}^{(k)}\right) \\
= & \frac{q^{k}}{1-q^{k}} \sum_{j=0}^{n} \frac{p^{j}}{j !}\left(\frac{1}{1-q^{k}}\right)^{(j)}+\frac{q^{2 k}}{\left(1-q^{k}\right)^{2}} \\
& -\frac{q^{k}}{1-q^{k}}\left[\sum_{j=0}^{n} \frac{p^{j}}{j !}\left(\frac{1}{1-q^{k}}\right)^{(j)}-1\right] \\
= & \frac{q^{k}}{\left(1-q^{k}\right)^{2}}=\operatorname{Var}\left(R_{0}^{(k)}\right) .
\end{aligned}
$$

The last equality is a consequence of (2.5).

Recurrence relations (3.5) and (3.6) can be further simplified for $k=1$ and $k=2$. An easy computation shows that if $k=1$ then we get

$$
\mathrm{E}\left(R_{m} R_{n}\right)=\frac{(n+q)(m+q)+(m+1) q}{p^{2}}, \quad \operatorname{Cov}\left(R_{m}, R_{n}\right)=\frac{(m+1) q}{p^{2}}, \quad 0 \leq m<n .
$$

Note that the above conclusion can be also derived by use of the well-known representation $R_{n} \stackrel{\mathrm{d}}{=} \sum_{j=1}^{n+1} X_{j}+n$, where $X_{1}, X_{2}, \ldots$ are original iid $g e(p)$ rv's; see Arnold et al. (1998).

Setting $k=2$ and $m=0$ in (3.5) and (3.6) yields

$$
\begin{aligned}
\mathrm{E}\left(R_{0}^{(2)} R_{n}^{(2)}\right) & =\frac{q^{2}}{1-q^{2}} \sum_{j=0}^{n} \frac{p^{j}}{j !}\left(\frac{1}{1-q^{2}}\right)^{(j)}+\frac{q^{4}}{\left(1-q^{2}\right)^{2}}, \\
\operatorname{Cov}\left(R_{0}^{(2)}, R_{n}^{(2)}\right) & =\operatorname{Var}\left(R_{0}^{(2)}\right)=\frac{q^{2}}{\left(1-q^{2}\right)^{2}} .
\end{aligned}
$$

By the identity

$$
\left(\frac{1}{1-q^{2}}\right)^{(j)}=\frac{j !}{2}\left[\frac{1}{p^{j+1}}+\frac{(-1)^{j}}{(q+1)^{j+1}}\right],
$$

after some algebra, we obtain

$$
\mathrm{E}\left(R_{0}^{(2)} R_{n}^{(2)}\right)=\frac{q^{2}}{1-q^{2}}\left[\frac{n+1}{2 p}+\frac{1}{4}\left(1-\left(\frac{q-1}{1+q}\right)^{n+1}\right)+\frac{q^{2}}{1-q^{2}}\right]
$$

Remark 1 Note that (3.6) implies that for the geometric distribution we have

$$
\rho\left(R_{0}^{(k)}, R_{n}^{(k)}\right)=\sqrt{\operatorname{Var} R_{0}^{(k)} / \operatorname{Var} R_{n}^{(k)}}, \quad n, k=1,2, \ldots
$$




\subsection{Weak $k$ th records}

The following theorem is analogous to Theorem 1 and may be proved in much the same way with the convention that $W_{-1}^{(k)}=0$ for $k \geq 1$, so its proof is omitted.

Theorem 3 For any $k=1,2, \ldots$, and $0 \leq m<n$ :

$$
\begin{aligned}
\mathrm{E}\left(W_{m}^{(k)} W_{n}^{(k)}\right)=\mathrm{E} & \left(W_{m-1}^{(k)} W_{n}^{(k)}\right) \\
& +\sum_{m_{1}=0}^{\infty} \mathrm{P}\left(M_{m_{1}}^{(k)}=m\right) \sum_{m_{2}=m_{1}}^{\infty} \sum_{j=0}^{n-m} \mathrm{P}\left(M_{m_{2}}^{(k)}-M_{m_{1}}^{(k)}=j\right) \\
& +\sum_{m_{1}=0}^{\infty} m_{1} \mathrm{P}\left(M_{m_{1}}^{(k)}=m\right) .
\end{aligned}
$$

Theorem 3 and formula (2.3) yield the following recurrence relations for covariances between weak $k$ th records.

Corollary 2 For any $k=1,2, \ldots$, and $0 \leq m<n$ :

$$
\begin{aligned}
\operatorname{Cov}\left(W_{m}^{(k)}, W_{n}^{(k)}\right)= & \operatorname{Cov}\left(W_{m-1}^{(k)}, W_{n}^{(k)}\right) \\
& +\sum_{m_{1}=0}^{\infty} \mathrm{P}\left(M_{m_{1}}^{(k)}=m\right) \sum_{m_{2}=m_{1}}^{\infty} \sum_{j=0}^{n-m} \mathrm{P}\left(M_{m_{2}}^{(k)}-M_{m_{1}}^{(k)}=j\right) \\
& +\sum_{m_{1}=0}^{\infty}\left(m_{1}-\mathrm{E}\left(W_{n}^{(k)}\right)\right) \mathrm{P}\left(M_{m_{1}}^{(k)}=m\right) .
\end{aligned}
$$

The next result shows, that the conclusion of Theorem 3 and Corollary 2 can be simplified in the geometric case.

Theorem 4 Assume that the parent distribution is geometric ge $(p)$. Then for $k=$ $1,2, \ldots$ and $0 \leq m<n$ :

$$
\begin{aligned}
\mathrm{E}\left(W_{m}^{(k)} W_{n}^{(k)}\right)= & \mathrm{E}\left(W_{m-1}^{(k)} W_{n}^{(k)}\right)+q \frac{p^{m}}{m !}\left(\frac{q^{k+m-1}}{1-q^{k}}\right)^{(m)} \\
& \times\left[q \sum_{j=1}^{n-m} \frac{p^{j}}{j !}\left(\frac{q^{k+j-1}}{1-q^{k}}\right)^{(j)}+\frac{1}{1-q^{k}}\right] \\
& +\frac{q}{k} \frac{p^{m}}{m !}\left[(1-k)\left(\frac{q^{k+m-1}}{1-q^{k}}\right)^{(m)}+q\left(\frac{q^{k+m-1}}{1-q^{k}}\right)^{(m+1)}\right]
\end{aligned}
$$


and

$$
\begin{aligned}
& \operatorname{Cov}\left(W_{m}^{(k)}, W_{n}^{(k)}\right)=\operatorname{Cov}\left(W_{m-1}^{(k)}, W_{n}^{(k)}\right)+q \frac{p^{m}}{m !}\left(\frac{q^{k+m-1}}{1-q^{k}}\right)^{(m)} \\
& \times\left[1-q \sum_{j=n-m+1}^{n} \frac{p^{j}}{j !}\left(\frac{q^{k+j-1}}{1-q^{k}}\right)^{(j)}\right] \\
& +\frac{q}{k} \frac{p^{m}}{m !}\left[(1-k)\left(\frac{q^{k+m-1}}{1-q^{k}}\right)^{(m)}+q\left(\frac{q^{k+m-1}}{1-q^{k}}\right)^{(m+1)}\right]
\end{aligned}
$$

Proof In the geometric case, the rv $M_{m_{1}}^{(k)}$ is a sum of iid rv's $\xi_{i}^{(k)}, i=0,1, \ldots, m_{1}$. Notice that $M_{m_{1}}^{(k)}$ has a negative binomial distribution $n b\left(k\left(m_{1}+1\right), 1-p\right)$, because $\xi_{i}$ has a negative binomial distribution $n b(k, 1-p)$. With the convention $\left(\begin{array}{l}a \\ b\end{array}\right)=0$ for $a<b$, it follows that

$$
\begin{aligned}
\sum_{m_{1}=0}^{\infty} m_{1} \mathrm{P}\left(M_{m_{1}}^{(k)}=m\right)= & \sum_{m_{1}=0}^{\infty} m_{1}\left(\begin{array}{c}
\left(m_{1}+1\right) k+m-1 \\
m
\end{array}\right) q^{\left(m_{1}+1\right) k} p^{m} \\
= & p^{m} \frac{q}{k}\left[\sum_{m_{1}=1}^{\infty} m_{1} k\left(\begin{array}{c}
m_{1} k+m-1 \\
m
\end{array}\right) q^{m_{1} k-1}\right. \\
& \left.-\frac{k}{q} \sum_{m_{1}=1}^{\infty}\left(\begin{array}{c}
m_{1} k+m-1 \\
m
\end{array}\right) q^{m_{1} k}\right] .
\end{aligned}
$$

Using the formulas

$$
\sum_{m_{1}=1}^{\infty}\left(\begin{array}{c}
m_{1} k+m-1 \\
m
\end{array}\right) q^{m_{1} k}=\frac{q}{m !}\left(\sum_{m_{1}=1}^{\infty} q^{m_{1} k+m-1}\right)^{(m)}=\frac{q}{m !}\left(\frac{q^{k+m-1}}{1-q^{k}}\right)^{(m)}
$$

and

$$
\begin{aligned}
\sum_{m_{1}=1}^{\infty} m_{1} k\left(\begin{array}{c}
m_{1} k+m-1 \\
m
\end{array}\right) q^{m_{1} k-1} & =\left(\frac{q}{m !}\left(\frac{q^{k+m-1}}{1-q^{k}}\right)^{(m)}\right)^{(1)} \\
& =\frac{1}{m !}\left(\frac{q^{k+m-1}}{1-q^{k}}\right)^{(m)}+\frac{q}{m !}\left(\frac{q^{k+m-1}}{1-q^{k}}\right)^{(m+1)},
\end{aligned}
$$


for $m \geq 0$, we get

$$
\sum_{m_{1}=0}^{\infty} m_{1} \mathrm{P}\left(M_{m_{1}}^{(k)}=m\right)=\frac{q}{k} \frac{p^{m}}{m !}\left[(1-k)\left(\frac{q^{k+m-1}}{1-q^{k}}\right)^{(m)}+q\left(\frac{q^{k+m-1}}{1-q^{k}}\right)^{(m+1)}\right]
$$

Further, since $M_{m_{2}}^{(k)}-M_{m_{1}}^{(k)}$ has a negative binomial distribution $n b\left(\left(m_{2}-m_{1}\right) k, 1-p\right)$ and by (3.13), we obtain

$$
\begin{aligned}
\sum_{m_{2}=m_{1}}^{\infty} \sum_{j=0}^{n-m} \mathrm{P}\left(M_{m_{2}}^{(k)}-M_{m_{1}}^{(k)}=j\right) & =\sum_{j=1}^{n-m} p^{j} \sum_{m_{2}=0}^{\infty}\left(\begin{array}{c}
m_{2} k+j-1 \\
j
\end{array}\right) q^{m_{2} k}+\sum_{m_{2}=0}^{\infty} q^{m_{2} k} \\
& =q \sum_{j=1}^{n-m} \frac{p^{j}}{j !}\left(\frac{q^{k+j-1}}{1-q^{k}}\right)^{(j)}+\frac{1}{1-q^{k}}
\end{aligned}
$$

Hence

$$
\begin{aligned}
\sum_{m_{1}=0}^{\infty} \mathrm{P}\left(M_{m_{1}}^{(k)}=m\right) & \sum_{m_{2}=m_{1}}^{\infty} \sum_{j=0}^{n-m} \mathrm{P}\left(M_{m_{2}}^{(k)}-M_{m_{1}}^{(k)}=j\right)=p^{m} \sum_{m_{1}=1}^{\infty}\left(\begin{array}{c}
m_{1} k+m-1 \\
m
\end{array}\right) q^{m_{1} k} \\
& \times \sum_{m_{2}=m_{1}}^{\infty} \sum_{j=0}^{n-m} \mathrm{P}\left(M_{m_{2}}^{(k)}-M_{m_{1}}^{(k)}=j\right) \\
= & q \frac{p^{m}}{m !}\left(\frac{q^{k+m-1}}{1-q^{k}}\right)^{(m)}\left[q \sum_{j=1}^{n-m} \frac{p^{j}}{j !}\left(\frac{q^{k+j-1}}{1-q^{k}}\right)^{(j)}+\frac{1}{1-q^{k}}\right],
\end{aligned}
$$

where the last equality is a consequence of (3.13). Combining it with (3.14) and Theorem 3 we obtain (3.11). Moreover, analogously to the proof of (3.6), formulas (3.11) and (2.4) imply immediately (3.12). In particular, for $m=0$ we get $\operatorname{Cov}\left(W_{0}^{(k)}, W_{n}^{(k)}\right)=\operatorname{Var}\left(W_{0}^{(k)}\right)$.

We can simplify recurrence relations (3.11) and (3.12) for $k=1$ and $k=2$. When $k=1$, simple algebra yields

$\mathrm{E}\left(W_{m} W_{n}\right)=\frac{q(m+1)[q(n+1)+1]}{p^{2}}, \quad \operatorname{Cov}\left(W_{m}, W_{n}\right)=\frac{(m+1) q}{p^{2}}, \quad 0 \leq m<n$

Note that the above expressions can be also derived by use of the well-known representation $W_{n} \stackrel{\mathrm{d}}{=} \sum_{j=1}^{n+1} X_{j}$, where $X_{1}, X_{2}, \ldots$ are original iid $g e(p)$ rv's; see e.g. Dembińska (2007b). 
Setting $k=2$ and $m=0$ in (3.11) and (3.12), we obtain

$$
\begin{aligned}
\mathrm{E}\left(W_{0}^{(2)} W_{n}^{(2)}\right) & =\frac{q^{2}}{1-q^{2}}\left[q \sum_{j=1}^{n} \frac{p^{j}}{j !}\left(\frac{q^{j+1}}{1-q^{2}}\right)^{(j)}+\frac{1}{1-q^{2}}\right]+\frac{q^{4}}{\left(1-q^{2}\right)^{2}}, \\
\operatorname{Cov}\left(W_{0}^{(2)}, W_{n}^{(2)}\right) & =\operatorname{Var}\left(W_{0}^{(k)}\right)=\frac{q^{2}}{\left(1-q^{2}\right)^{2}} .
\end{aligned}
$$

Observe that for odd $j$ :

$$
\left(\frac{q^{j+1}}{1-q^{2}}\right)^{(j)}=\left(q^{j+1}+q^{j+3}+\cdots\right)^{(j)}=\left(1+q^{2}+q^{4}+\cdots\right)^{(j)}=\left(\frac{1}{1-q^{2}}\right)^{(j)}
$$

while for even $j$ :

$$
\left(\frac{q^{j+1}}{1-q^{2}}\right)^{(j)}=\left(q+q^{3}+q^{5}+\cdots\right)^{(j)}=\left(\frac{q}{1-q^{2}}\right)^{(j)} .
$$

Combining the above relations with (3.10) and

$$
\left(\frac{q}{1-q^{2}}\right)^{(j)}=\frac{j !}{2}\left[\frac{(-1)^{j+1}}{(q+1)^{j+1}}+\frac{1}{(1-q)^{j+1}}\right],
$$

we get for $j \geq 0$ :

$$
\frac{p^{j}}{j !}\left(\frac{q^{j+1}}{1-q^{2}}\right)^{(j)}=\frac{1}{2 p}\left[1-\left(\frac{p}{2-p}\right)^{j+1}\right]
$$

which, after some algebra, gives

$$
\mathrm{E}\left(W_{0}^{(2)} W_{n}^{(2)}\right)=\frac{q^{2}}{1-q^{2}}\left[\frac{n q+1}{2 p}+\frac{1}{4}\left(1+\left(\frac{p}{2-p}\right)^{n+1}\right)\right]+\frac{q^{4}}{\left(1-q^{2}\right)^{2}} .
$$

Remark 2 Note that (3.12) implies that for the geometric distribution we have

$$
\rho\left(W_{0}^{(k)}, W_{n}^{(k)}\right)=\sqrt{\operatorname{Var} W_{0}^{(k)} / \operatorname{Var} W_{n}^{(k)}}, \quad n, k=1,2 \ldots
$$

\subsection{Strong $k$ th records}

By Lemma 1, to derive expressions for the product moments of strong $k$ th records from cdf $F$, it suffices to find the corresponding ones of records from cdf $F_{1: k}$ :

$$
\mathrm{E}\left(S_{m}^{(k)}(F) S_{n}^{(k)}(F)\right)=\mathrm{E}\left(R_{m}\left(F_{1: k}\right) R_{n}\left(F_{1: k}\right)\right)
$$


This relation enables us to obtain easily the desired formulas for geometric strong $k$ th records. Formulas given in (3.9) with the fact that $F_{1: k}$ is geometric $g e\left(1-q^{k}\right)$ provided that $F$ is geometric $g e(p)$, imply the following theorem.

Theorem 5 For any $0 \leq m<n$ and $k=1,2, \ldots$ :

$\mathrm{E}\left(S_{m}^{(k)} S_{n}^{(k)}\right)=\frac{\left(n+q^{k}\right)\left(m+q^{k}\right)+(m+1) q^{k}}{\left(1-q^{k}\right)^{2}}, \quad \operatorname{Cov}\left(S_{m}^{(k)}, S_{n}^{(k)}\right)=\frac{(m+1) q^{k}}{\left(1-q^{k}\right)^{2}}$

Moreover, it is known that the correlation coefficient between geometric records $R_{m}$ and $R_{n}$ is equal to $\sqrt{(m+1) /(n+1)}, m<n$ (see, for example, Arnold et al. 1998 , p. 40). This immediately implies that in the geometric case we get

$$
\rho\left(S_{m}^{(k)}, S_{n}^{(k)}\right)=\sqrt{\frac{m+1}{n+1}}, \quad 0 \leq m<n .
$$

Table 1 Correlation coefficients between geometric ge $(p=0.25)$ strong 2 nd records $S_{m}^{(2)}$ and $S_{n}^{(2)}$, 2nd records $R_{m}^{(2)}$ and $R_{n}^{(2)}$ (in brackets) and weak 2 nd records $W_{m}^{(2)}$ and $W_{n}^{(2)}$ (in square brackets)

\begin{tabular}{lllllll}
\hline & $m=0$ & $m=1$ & $m=2$ & $m=3$ & $m=4$ & $m=5$ \\
\hline$n=1$ & 0.707107 & 1.000000 & - & - & - & - \\
& $(0.694239)$ & $(1.000000)$ & - & - & - & - \\
& {$[0.698257]$} & {$[1.000000]$} & - & - & - & - \\
$n=2$ & 0.577350 & 0.816497 & 1.000000 & - & - & - \\
& $(0.569690)$ & $(0.808298)$ & $(1.000000)$ & - & - & - \\
& {$[0.569690]$} & {$[0.810657]$} & {$[1.000000]$} & - & - & - \\
$n=3$ & 0.500000 & 0.707107 & 0.866025 & 1.000000 & - & - \\
& $(0.493559)$ & $(0.701801)$ & $(0.859939)$ & $(1.000000)$ & - & - \\
& {$[0.493617]$} & {$[0.701760]$} & {$[0.861647]$} & {$[1.000000]$} & - & - \\
$n=4$ & 0.447213 & 0.632456 & 0.774597 & 0.894427 & 1.000000 & - \\
& $(0.441711)$ & $(0.627884)$ & $(0.770425)$ & $(0.889592)$ & $(1.000000)$ & - \\
& {$[0.441711]$} & {$[0.627884]$} & {$[0.770425]$} & {$[0.890916]$} & {$[1.000000]$} & - \\
$n=5$ & 0.408248 & 0.573504 & 0.707107 & 0.816497 & 0.912871 & 1.000000 \\
& $(0.403360)$ & $(0.573395)$ & $(0.703428)$ & $(0.813055)$ & $(0.908856)$ & $(1.000000)$ \\
& {$[0.403361]$} & {$[0.573360]$} & {$[0.703456]$} & {$[0.813054]$} & {$[0.909939]$} & {$[1.000000]$} \\
$n=6$ & 0.377964 & 0.534522 & 0.654654 & 0.755929 & 0.845154 & 0.925820 \\
& $(0.373532)$ & $(0.530989)$ & $(0.651423)$ & $(0.752837)$ & $(0.842218)$ & $(0.922387)$ \\
& {$[0.373532]$} & {$[0.530958]$} & {$[0.651423]$} & {$[0.752860]$} & {$[0.842218]$} & {$[0.923302]$} \\
$n=7$ & 0.353553 & 0.500000 & 0.612372 & 0.707107 & 0.790569 & 0.866025 \\
& $(0.349472)$ & $(0.496788)$ & $(0.609462)$ & $(0.704358)$ & $(0.787893)$ & $(0.863463)$ \\
& {$[0.349472]$} & {$[0.496758]$} & {$[0.609463]$} & {$[0.704357]$} & {$[0.787912]$} & {$[0.863463]$} \\
\hline \multirow{5}{*}{$n=1$} & & & &
\end{tabular}


Table 2 Correlation coefficients between geometric ge $(p=0.5)$ strong 2 nd records $S_{m}^{(2)}$ and $S_{n}^{(2)}$, 2nd records $R_{m}^{(2)}$ and $R_{n}^{(2)}$ (in brackets) and weak 2 nd records $W_{m}^{(2)}$ and $W_{n}^{(2)}$ (in square brackets)

\begin{tabular}{lllllll}
\hline & $m=0$ & $m=1$ & $m=2$ & $m=3$ & $m=4$ & $m=5$ \\
\hline$n=1$ & 0.707107 & 1.000000 & - & - & - & - \\
& $(0.625543)$ & $(1.000000)$ & - & - & - & - \\
& {$[0.670820]$} & {$[1.000000]$} & - & - & - & - \\
$n=2$ & 0.577350 & 0.816497 & 1.000000 & - & - & - \\
& $(0.539784)$ & $(0.762858)$ & $(1.000000)$ & - & - & - \\
& {$[0.539784]$} & {$[0.791252]$} & {$[1.000000]$} & - & - & - \\
$n=3$ & 0.500000 & 0.707107 & 0.866025 & 1.000000 & - & - \\
& $(0.462367)$ & $(0.682012)$ & $(0.823711)$ & $(1.000000)$ & - & - \\
& {$[0.466072]$} & {$[0.679339]$} & {$[0.846876]$} & {$[1.000000]$} & - & - \\
$n=4$ & 0.447214 & 0.632456 & 0.774597 & 0.894427 & 1.000000 & - \\
& $(0.417022)$ & $(0.606539)$ & $(0.752810)$ & $(0.861020)$ & $(1.000000)$ & - \\
& {$[0.417022]$} & {$[0.606694]$} & {$[0.752810]$} & {$[0.878940]$} & {$[1.000000]$} & - \\
$n=5$ & 0.408248 & 0.573504 & 0.707107 & 0.816497 & 0.912871 & 1.000000 \\
& $(0.380782)$ & $(0.577350)$ & $(0.684382)$ & $(0.798647)$ & $(0.884958)$ & $(1.000000)$ \\
& {$[0.381123]$} & {$[0.554117]$} & {$[0.686500]$} & {$[0.798458]$} & {$[0.899834]$} & {$[1.000000]$} \\
$n=6$ & 0.377964 & 0.534522 & 0.654654 & 0.755929 & 0.845154 & 0.925820 \\
& $(0.353262)$ & $(0.515419)$ & $(0.635849)$ & $(0.737076)$ & $(0.829701)$ & $(0.901952)$ \\
& {$[0.353262]$} & {$[0.513500]$} & {$[0.635849]$} & {$[0.738599]$} & {$[0.829701]$} & {$[0.914551]$} \\
$n=7$ & 0.353553 & 0.500000 & 0.612372 & 0.707107 & 0.790569 & 0.866025 \\
& $(0.330743)$ & $(0.482815)$ & $(0.595026)$ & $(0.691292)$ & $(0.774096)$ & $(0.852500)$ \\
& {$[0.330776]$} & {$[0.480781]$} & {$[0.595231]$} & {$[0.691121]$} & {$[0.775532]$} & {$[0.852485]$} \\
\hline \multirow{5}{*}{$n=1$} & & & & &
\end{tabular}

\section{Tables}

In this section, we present Tables 1, 2, 3 with numerical values of correlation coefficients between strong 2nd records $S_{m}^{(2)}$ and $S_{n}^{(2)}$, 2nd records $R_{m}^{(2)}$ and $R_{n}^{(2)}$ and weak 2nd records $W_{m}^{(2)}$ and $W_{n}^{(2)}$ for the geometric parent $g e(p)$, where $m<n$ and $m=0,1, \ldots, 5, n=1, \ldots, 7$ and $p=0.25,0.5,0.75$. They provide us some insight into their magnitude of change. It is worth pointing out that in all the presented cases the correlation coefficients between 2 nd records $R_{m}^{(2)}$ and $R_{n}^{(2)}$ as well as between weak 2 nd records $W_{m}^{(2)}$ and $W_{n}^{(2)}$ do not exceed the correlation coefficients of 2 nd strong records $S_{m}^{(2)}$ and $S_{n}^{(2)}$ given in (3.15), which is also the maximum correlation possible between any continuous square-integrable $k$ th records (see, Nevzorov 1992). 
Table 3 Correlation coefficients between geometric ge $(p=0.75)$ strong 2 nd records $S_{m}^{(2)}$ and $S_{n}^{(2)}$, 2nd records $R_{m}^{(2)}$ and $R_{n}^{(2)}$ (in brackets) and weak 2 nd records $W_{m}^{(2)}$ and $W_{n}^{(2)}$ (in square brackets)

\begin{tabular}{|c|c|c|c|c|c|c|}
\hline & $m=0$ & $m=1$ & $m=2$ & $m=3$ & $m=4$ & $m=5$ \\
\hline \multirow[t]{3}{*}{$n=1$} & 0.707107 & 1.000000 & - & - & - & - \\
\hline & $(0.436852)$ & $(1.000000)$ & - & - & - & - \\
\hline & {$[0.627456]$} & [1.000000] & - & - & - & - \\
\hline \multirow[t]{3}{*}{$n=2$} & 0.577350 & 0.816497 & 1.000000 & - & - & - \\
\hline & $(0.481660)$ & $(0.617777)$ & $(1.000000)$ & - & - & - \\
\hline & [0.481660] & {$[0.756760]$} & [1.000000] & - & - & - \\
\hline \multirow[t]{3}{*}{$n=3$} & 0.500000 & 0.707107 & 0.866025 & 1.000000 & - & - \\
\hline & $(0.351665)$ & $(0.663417)$ & $(0.667668)$ & (1.000000) & - & - \\
\hline & [0.403497] & [0.628485] & [0.819809] & {$[1.000000]$} & - & - \\
\hline \multirow[t]{3}{*}{$n=4$} & 0.447213 & 0.632456 & 0.774597 & 0.894427 & 1.000000 & - \\
\hline & $(0.354505)$ & $(0.540323)$ & $(0.710828)$ & $(0.738767)$ & (1.000000) & - \\
\hline & [0.354505] & [0.549293] & [0.710828] & [0.856947] & [1.000000] & - \\
\hline \multirow[t]{3}{*}{$n=5$} & 0.408248 & 0.573504 & 0.707107 & 0.816497 & 0.912871 & 1.000000 \\
\hline & $(0.304617)$ & $(0.530510)$ & $(0.591323)$ & $(0.773648)$ & $(0.771311)$ & $(1.000000)$ \\
\hline & [0.320632] & [0.495245] & [0.637786] & {$[0.763325]$} & [0.881305] & [1.000000] \\
\hline \multirow[t]{3}{*}{$n=6$} & 0.377964 & 0.534522 & 0.654653 & 0.755929 & 0.845154 & 0.925820 \\
\hline & $(0.295549)$ & $(0.476165)$ & $(0.585056)$ & $(0.669791)$ & $(0.799557)$ & $(0.806101)$ \\
\hline & [0.295549] & {$[0.455635]$} & {$[0.585056]$} & [0.697115] & [0.799557] & [0.898473] \\
\hline \multirow[t]{3}{*}{$n=7$} & 0.353553 & 0.500000 & 0.612372 & 0.707107 & 0.790569 & 0.866025 \\
\hline & $(0.270859)$ & $(0.457585)$ & $(0.529947)$ & $(0.658281)$ & $(0.7046041)$ & $(0.829010)$ \\
\hline & [0.276009] & {$[0.425025]$} & {$[0.544786]$} & [0.647385] & {$[0.739519]$} & [0.826016] \\
\hline
\end{tabular}

\section{Compliance with ethical standards}

Conflict of interest On behalf of all authors, the corresponding author states that there is no conflict of interest.

Open Access This article is distributed under the terms of the Creative Commons Attribution 4.0 International License (http://creativecommons.org/licenses/by/4.0/), which permits unrestricted use, distribution, and reproduction in any medium, provided you give appropriate credit to the original author(s) and the source, provide a link to the Creative Commons license, and indicate if changes were made.

\section{References}

Ahsanullah M, Aliev F (2011) A characterization of geometric distribution based on weak records. Stat Probab Lett 52:651-655

Arnold BC, Balakrishnan N, Nagaraja HN (1998) Records. Wiley, New York

Block HW, Fang Z (1988) A multivariate extension of Hoeffding's lemma. Ann Probab 16:1803-1820

Charalambides ChA, Rychlik T (2008) Distributions and moments of record values in a sequence of maximally dependent random variables. J Stat Plann Inference 138:2253-2266

Danielak K, Dembińska A (2007a) Some characterizations of discrete distributions based on weak records. Stat Pap 48:479-489 
Danielak K, Dembińska A (2007b) On characterizing discrete distributions via conditional expectations of weak record values. Metrika 66:129-138

Dembińska A (2007a) A review on characterizations of discrete distributions based on records and kth records. Commun Stat Theory Methods 36:1381-1387

Dembińska A (2007b) Characterizations of discrete distributions based on record values. In: Ahsanullah M, Kirmani S (eds) Extreme Value Distributions. Nova Science Publishers, New York, pp 63-75

Dembińska A (2008) $k$ th records from geometric distribution. Stat Probab Lett 78:1662-1670

Dembińska A, Danielak K (2008) On moments of $k$-records from discrete distributions. Commun Stat Theory Methods 37:2516-2531

Dembińska A, López-Blázquez F (2005a) A characterization of geometric distribution through $k$ th weak records. Commun Stat Theory Methods 34:2345-2351

Dembińska A, López-Blázquez F (2005b) $k$ th records from discrete distributions. Stat Probab Lett 71:203214

Dembińska A, Stepanov A (2006) Limit theorems for the ratio of weak records. Stat Probab Lett 76:14541464

Goroncy A, Rychlik T (2011) Lower bounds on the expectations of upper record values. J Stat Plann Inference 141:2726-2737

Hoeffding W (1940) Maßtabinvariante Korrelations - Theorie. Schrift Math Inst Univ Berlin 5:181-233

Jasiński K (2016) On maximum variance of kth records. Commun Stat Theory Methods 45:2392-2401

Karczewski R, Wesołowski J (2017) Linearity of regression for weak records, revisited. Statistics 51:878887

Klimczak M, Rychlik T (2004) Maximum variance of $k$ th records. Stat Probab Lett 69:421-430

Klimczak M, Rychlik T (2005) Recontruction of previous failure times and records. Metrika 61:277-290

Kozyra PM, Rychlik T (2017) Sharp bounds on the expectations of linear combinations of $k$ th records expressed in the Gini mean difference units. Probab Math Stat Pol (to appear)

López-Blázquez F, Salamanca Miño B, Dembińska A (2005) A note on the distribution of $k$ th records from discrete distributions. Stat Probab Lett 75:325-330

Nevzorov VB (1992) A characterization of exponential distributions by correlation between records. Math Meth Stat 1:49-54

Nevzorov VB (2001) Records: mathematical theory, translation of mathematical monographs, vol 194. American Mathematical Society, Providence

Oncel SY, Aliev F (2016) Characterizations of geometric and discrete pareto distributions based on the conditional distribution of $k$ th records. J Stat Theory Appl 15:367-372

Raqab MZ, Rychlik T (2002) Sharp bounds for the mean of $k$ th record value. Commun Stat Theory Methods 31:1927-1937

Raqab MZ, Rychlik T (2004) Sharp bounds on the expectations of second record values from symmetric populations. Statistics 38:49-57

Raqab MZ, Rychlik T (2011) Bounds for the mean residual life function of a $k$-out-of- $n$ system. Metrika 74:361-380

Stepanov AV (1992) Limit theorems for weak records. Theory Probab Appl 37:570-574 (English translation)

Stepanov AV (1993) A characterization theorem for weak records. Theory Probab Appl 38:762-764 (English translation)

Stepanov AV, Balakrishnan N, Hofmann G (2003) Exact distribution and Fisher information of weak record values. Stat Probab Lett 64:69-81

Vervaat W (1973) Limit theorems for records from discrete distributions. Stoch Process Their Appl 1:317334

Wesołowski J, Ahsanullah M (2001) Linearity of regression for non-adjacent weak records. Stat Sin 11(1):39-52 